Artigo

\title{
Modelagem dos Recursos Eólicos sobre Moçambique Considerando um Cenário de Mudanças Climáticas
}

\author{
Emerson Mariano da Silva ${ }^{1}$, Nelson Mário Banga ${ }^{2}$, José Maria Brabo Alves ${ }^{1}$ \\ ${ }^{1}$ Universidade Estadual do Ceará, Fortaleza, CE, Brasil. \\ ${ }^{2}$ Faculdade de Engenharia Ambiental e de Recursos Naturais, Uni Zambeze, Beira, \\ Mozambique.
}

Recebido: 6 de Agosto de 2015 - Aceito: 8 de Fevereiro de 2017

\begin{abstract}
Resumo
Este trabalho apresenta uma aplicação da técnica de redução de escala (dynamic downscaling) usando-se o modelo regional RAMS6.0 (Regional Atmospheric Modelling System) forçado por campos de saída do modelo de circulação global HadGEM2-ES, com objetivo de estimar o potencial eólico nas regiões de Moçambique considerando um cenário de mudanças climáticas definido pelo IPCC. Os resultados mostraram desempenho satisfatório do modelo RAMS6.0 na reprodução de padrões médios de campos dos ventos obtidos nas reanálises do CFSR e MERRA durante o período de 1985-2005. Os campos de ventos simulados a 10, 50 e $100 \mathrm{~m}$ de altura, sob condições do cenário de emissão RCP8.5 do IPCC, para o período de 2079-2099, mostraram-se com valores superiores em relação aos campos de ventos para o período de 1985-2005. Além disso, há evidências de um aumento na densidade de energia eólica nas regiões de Moçambique para o período de 2079-2099, particularmente durante o inverno nas regiões centro e sul do país.
\end{abstract}

Palavras chave: energia eólica, mudanças climáticas, Moçambique.

\section{Modeling of Wind Resources on Mozambique Considering a Climate Change Scenario}

\begin{abstract}
This work presents the application of the dynamic downscaling technique on the Regional Atmospheric Modeling System (RAMS6.0) forced by output fields of the global circulation model HadGEM2-ES, to estimate the wind potential over the regions of Mozambique considering the climate change scenario defined by IPCC. The results showed satisfactory performance of the RAMS6.0 model in the reproduction of mean patterns of wind fields obtained in the reanalysis compared to CFSR and MERRA during the period 1985-2005. Winds fields at 10, 50 and 100 meters Considering conditions of the IPCC emission scenario (RCP8.5) for the period 2079-2099 showed higher values in relation to the wind fields for the period 1985-2005. Furthermore, there is evidence of an increase in the wind power over regions of Mozambique for the period 2079-2099, particularly during winter in the central and southern regions of the country.
\end{abstract}

Keywords: wind energy, climate change, Mozambique.

\section{Introdução}

A energia eólica é uma fonte de energia renovável que tem apresentado um crescimento acelerado no mundo, dada à contribuição que esta fonte pode desempenhar na provisão de energia no futuro, bem como pela sua contribuição na mitigação de alterações climáticas, pois é considerada uma das fontes de energia limpa, por apresentar baixa emissão de gases do efeito estufa (Oliveira et al., 2003).

A produção de energia eólica vem registrando crescimento acentuado desde os anos inicias do século XXI. De acordo com dados do Conselho Mundial de Energia Eólica (Global Wind Energy Council - GWEC, 2012), a capacidade acumulada de geração de energia eólica no mundo vem crescendo anualmente, como por exemplo em 2012

Autor de correspondência: Emerson Mariano da Silva, emerson@uece.br. 
que atingiu $282 \mathrm{GW}$, que representou um aumento de $19 \%$ em relação ao ano anterior.

O mercado de energia eólica na África tem evoluído nos últimos tempos, principalmente, pelas necessidades que são impostas aos demais estados africanos na geração de energia para atender a demanda existente e futura. Segundo o Conselho Mundial de Energia (2010), o continente africano possui potencial de energia renovável para atender as futuras necessidades da matriz energética local, estimase que no continente 18 dos 35 principais países em desenvolvimento apresentam alta classificação em reservas de energia renováveis (Buys et al., 2007).

Estudo realizado por Pinto (2008), no período de 1999 a 2003, utilizando o modelo atmosférico RegCM3, mostraram uma expectativa de significativos recursos eólicos sobre a região de Moçambique, com intensidade máxima dos ventos médios entre 7,0 e $9,0 \mathrm{~m} / \mathrm{s}$, no período de junho à agosto, ocorrendo na maior parte das regiões do litoral e planaltos moçambicanos.

$\mathrm{O}$ uso da energia eólica em Moçambique representa uma das alternativas para suprir a demanda energética futura do país, pois, atualmente observa-se uma redução na produção de energia elétrica, associada a diminuição da vazão do Rio Zambeze, devido a alta taxa de evapotranspiração induzida pelas mudanças climáticas (INGC, 2009).

Estudos dos impactos das mudanças climáticas evidenciam possíveis mudanças nos padrões de circulação atmosférica e na produção da energia eólica em várias partes do mundo (Harrison et al., 2008; Sailor et al., 2008; Breslow e Sailor, 2002). Segal et al. (2001) realizaram simulações climáticas usando o modelo atmosférico regional RegCM2 acoplado ao modelo de circulação geral HadCM2, forçado pela condição de fronteira do clima presente e por um cenário de aumento da concentração de CO2 na atmosfera (representado a década de 2040), que mostraram reduções sazonais em cerca de $30 \%$ na densidade de potencia eólica quando se tem o clima futuro com aumento da quantidade de $\mathrm{CO} 2$ na atmosfera.

Breslow e Sailor (2002) mostraram que os campos de vento de um modelo atmosférico de circulação geral do $\mathrm{Ca}$ nadian Centre for Climate Modelling and Analysis são susceptíveis a mudanças climáticas resultantes do aumento da concentração do $\mathrm{CO} 2$ atmosférico ao longo dos próximos 100 anos.

Pryor et al. (2005) apresentam resultados de simulações climáticas obtidos por cinco modelos globais em regiões no Norte da Europa, comparados com observações de 45 estações meteorológicas. Mostraram que na maioria das estações não existe diferença significativa entre as condições durante os períodos de 2046-2065 e 1961-1990, enquanto que, no período de 2081-2100 a média da velocidade do vento e a densidade de energia são ligeiramente menores em relação ao período de 1961-1990.

Sailor et al. (2008) usando a técnica de downscaling estatístico e os dados de saída de um modelo de circulação geral da atmosfera, investigaram o impacto do cenário das mudanças climáticas sobre a geração da energia eólica em cinco regiões do nordeste dos Estados Unidos. Verificaram redução de 5 a $10 \%$ na velocidade do vento durante o verão, mostrando um alto impacto das mudanças climáticas no potencial de geração de energia eólica no verão dessas regiões, equivalente a uma redução da ordem de $40 \%$.

Nolan et al. (2011) avaliaram o futuro dos recursos eólicos através de simulações climatológicas de alta resolução, usando o modelo climático regional RCM em regiões irlandesas. As simulações foram efetuadas para o período de controle de 1961 a 2000, e para um período futuro de 2021 a 2060. Assim, mostraram um aumento substancial no conteúdo da energia eólica para o futuro no inverno e um decréscimo durante os meses de verão, como também significativas estatísticas nas mudanças projetadas para o verão e inverno por toda a Irlanda.

Os estudos acima descritos indicam que as possíveis mudanças climáticas devem afetar de diferentes formas a produção energia eólica em várias partes do mundo. Assim, uma investigação desses impactos é importante para futuras projeções de produção de energia eólica na região de Moçambique, pois uma pequena variação nos padrões de velocidade do vento pode resultar em um aumento ou diminuição significativa do potencial de geração de energia eólica na região, já que a densidade de potência estimada é dependente dos valores da velocidade do vento elevados a terceira potência.

Face ao exposto, o principal objetivo do trabalho é estimar o potencial eólico sobre Moçambique no período futuro, através de simulações da climatologia dos ventos, usando modelagem atmosférica e considerando os cenários futuros de emissões definidos pelo Painel Intergovernamental sobre Mudanças Climáticas (IPCC), com vista a auxiliar os futuros projetos de aproveitamento dos recursos eólicos para geração de energia elétrica na região.

\section{Materiais e Métodos}

Na primeira etapa do trabalho foram realizadas simulações climáticas dos campos médios de velocidade dos ventos sobre Moçambique (Fig. 1) usando a técnica de redução de escala (dynamic downscaling) com o modelo regional RAMS6.0 (Pielke et al., 1992; Cotton et al., 1998) acoplado ao modelo global HadGEM2-ES.

Com o objetivo de avaliar o desempenho das simulações dos campos médios de velocidade de ventos sobre Moçambique foram usados duas bases de dados de reanálises. Os dados do CFSR (Climate Forecast System Reanalysis) descrito em Saha et al. (2010), resultantes de estimativas de satélite com uma resolução horizontal de $0,3^{\circ}$ de longitude por $0,3^{\circ}$ de latitude, contendo componentes de velocidade de vento a $10 \mathrm{~m}$ da superfície, e os dados do MERRA (Modern - ERA Retrospective Analysis for Research and Applications), descritos em Bosilovich et al. 


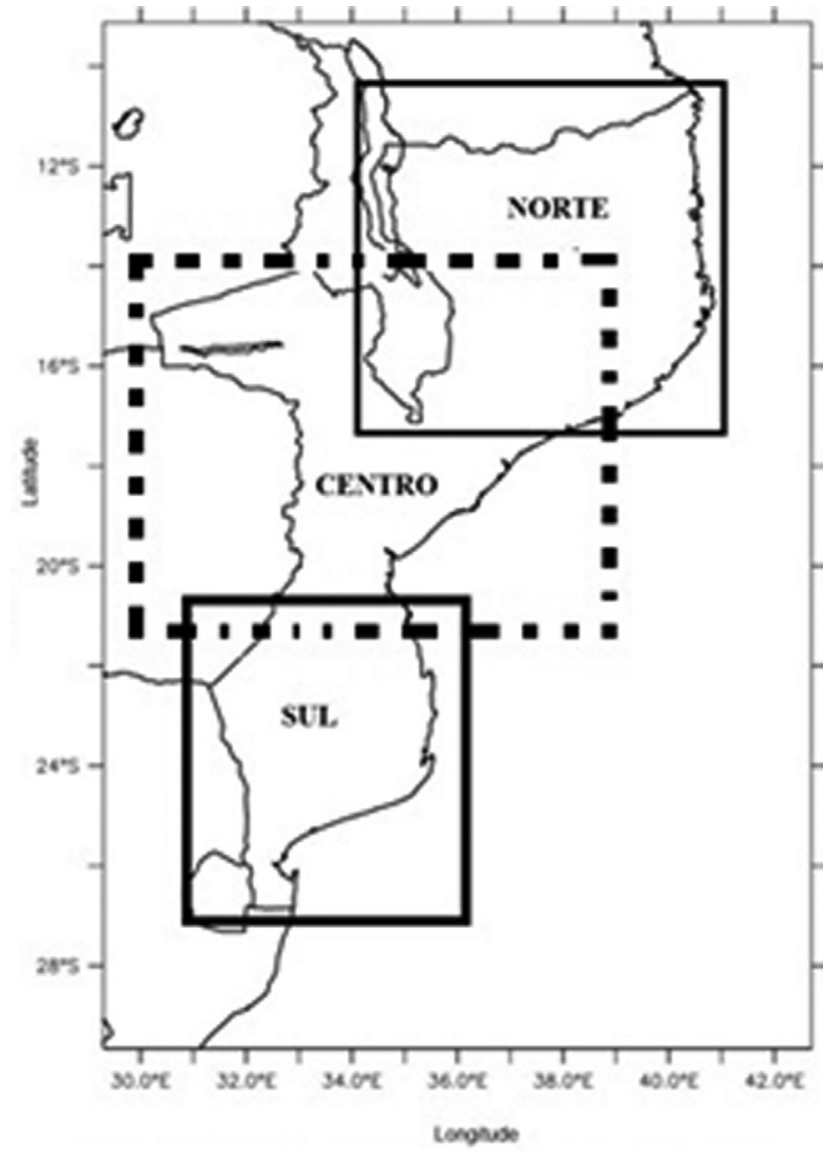

Figura 1 - Domínio de simulações com suas respectivas regiões de análise.

(2008), que são resultantes de estimativas de satélite com uma resolução horizontal de $0.6^{\circ}$ de longitude por $0.5^{\circ}$ de latitude.

Foram utilizados os índices estatísticos descritos em Santiago de Maria et al. (2008): Viés estatístico (b), Erro Quadrático Médio (EQM), coeficiente de correlação $(r)$ e o índice de concordância (Willmott, 1981), mostrados na Tabela 1.

$\mathrm{O}$ viés estatístico é definido como medida da média da diferença entre dois conjuntos de dados $X$ e $Y$, em que $X_{i}$ e $Y_{i}$ são as i-ésimas observações do conjunto de dados e $N$ é o número de observações.

O erro quadrático médio é definido como medida da diferença entre dois conjuntos de dados $X$ e $Y$. No entanto, se os conjuntos de dados $X$ e $Y$ estiverem em perfeita concordância, todos os valores $X_{i}$ e $Y_{i}$ são idênticos erro quadrático médio e o viés é igual à zero.

O coeficiente de correlação de Pearson $(r)$ descreve o grau de colinearidade entre os dados simulados e observados, indica a concordância entre diferentes conjuntos de dados. Sendo que, representa desvio padrão da variável simulada e representa desvio padrão da variável observada.

Para avaliar o nível de significância observada nas correlações entre os campos de ventos simulados com os das reanálises foi usado o teste t-Student (Huang e Paes, 2009; Menezes et al., 2008). O teste usado é dado pela expressão matemática:

$$
t=\frac{r \sqrt{N-2}}{\sqrt{1-r^{2}}}
$$

em que, $N$ é o número de pares de dados.

A partir da expressão (1) é extraído o coeficiente crítico de correlação $\left(r_{c}\right)$, que é o valor usado para aceitar ou rejeitar no teste de hipótese, sendo dado por:

$$
r_{c}=\sqrt{\frac{t^{2}}{(N-2)+t^{2}}}
$$

Neste trabalho foram usados 2250 pares de dados para analisar as correlações espaciais sobre a região norte, 3420 para região central e 1519 pares de dados para a região sul. Os coeficientes de correlação foram comparados aos coeficientes de correlação de Person, mostrados na Tabela 2 .

\begin{tabular}{|c|c|c|c|}
\hline Índice & Símbolo & Fórmula & Valor ideal \\
\hline Viés & $b$ & $b=\frac{1}{N} \sum_{i=1}^{N}\left(X_{i}-Y_{i}\right)$ & 0 \\
\hline Erro quadrático médio & $E Q M$ & $E Q M=\left[\frac{1}{N} \sum_{i=1}^{N}\left(X_{i}-Y_{i}\right)^{2}\right]^{\frac{1}{2}}$ & 0 \\
\hline Índice de Correlação & $r$ & $r=\frac{1}{N} \sum_{i=1}^{N} \frac{X_{i} Y_{i}}{\sigma_{X} \sigma_{Y}}$ & 1 \\
\hline Índice de concordância & $I_{c}$ & $I_{c}=1-\frac{\sum_{i=1}^{N}\left(X_{i}-Y_{i}\right)^{2}}{\sum_{i=1}^{N}\left(\left|X_{i}-\bar{X}_{i}\right|+\left|Y_{i}-\bar{X}_{i}\right|\right)^{2}}$ & 1 \\
\hline
\end{tabular}

Tabela 1 - índices estatísticos com seus respectivos símbolos e fórmulas. 
Tabela 2 - Classificação do coeficiente de correlação. Fonte: Devore (2006).

\begin{tabular}{lc}
\hline Faixa & Classificação \\
\hline 1 & Perfeita \\
0,7 a 0,9 & Forte \\
0,4 a 0,7 & Moderada \\
0,2 a 0,4 & Fraca \\
0 & Sem correlação \\
\hline
\end{tabular}

Tabela 3 - Classificação do índice de concordância.

\begin{tabular}{lc}
\hline Faixa & Classificação \\
\hline$>0,85$ & Ótimo \\
0,76 a 0,85 & Muito bom \\
0,66 a 0,75 & Bom \\
0,61 a 0,65 & Mediano \\
0,51 a 0,60 & Razoável \\
0,41 a 0,50 & Ruim \\
$<0,40$ & Péssimo \\
\hline
\end{tabular}

O índice de concordância é interpretado como a medida do quanto os erros livres do modelo prevê a variável, ou seja, o $I_{c}$ determina em que medida as magnitudes e sinais dos valores observados são relacionados com os desvios das previsões. O $I_{c}$ não é adimensional, no entanto, se os valores simulados ajustarem aos valores observados seu valor é igual a 1 (ver Tabela 2). Os índices de concordância obtidos foram classificados na Tabela 3.

Segundo Willmott (1981) a utilização deste índice é fundamental, pois o erro absoluto médio e o erro quadrático médio não informam o tamanho relativo da diferença sistemática, ou mesmo a diferença dessa diferença.

Na segunda etapa do trabalho foram realizadas simulações dos campos de ventos para o período de 2079-2099, considerando o cenário de emissão RCP8.5 do IPCC, com objetivo de avaliar as possíveis mudanças na velocidade dos ventos e na densidade de potência eólica sobre as regiões de Moçambique. A quantificação das mudanças ocorridas na velocidade do vento foram realizadas através do cálculo de diferenças entre as séries de dados dos dois períodos de análise, através da expressão matemática (3), mostrada abaixo, que permite identificar a magnitude das mudanças e as tendências de variação (negativa ou positiva).

Variação percentual $=[($ média futura -

média histórica)/média histórica $] \times 100$

\subsection{Configurações dos modelos HadGEM2-ES e RAMS6.0}

O RAMS (Regional Modeling Atmospheric System) é um modelo desenvolvido por pesquisadores da Colorado
State University (Pielke et al., 1992; Cotton et al., 1998), baseia-se em equações que representam os processos físicos da dinâmica e termodinâmica da atmosfera, com algumas parametrizações dos efeitos da turbulência, da radiação solar e terrestre, dos fluxos de calor latente e sensível, assim como da convecção observada na atmosfera (Tremback e Walko, 2005).

As simulações climáticas com o RAMS foram realizadas para o período denominado de histórico, dezembro de 1985 a novembro de 2005, com uma resolução espacial e horizontal de $15 \mathrm{~km}$, com 82 por 148 pontos de grade, forçado pelos campos de saída do modelo de circulação geral HadGEM2-ES. As demais configurações usadas nas simulações deste estudo estão descritas na Tabela 4.

O HadGEM2-ES (Hadley Centre Global Environmental Model version 2 - Earth System) desenvolvido pelo UK Met Office é um modelo acoplado usado no CIMP5 (Coupled Model Intercomparison Project 5) em simulações para investigar a evolução do clima futuro.

O modelo climático possui um modelo atmosférico de circulação geral com 38 níveis verticais (chegando até aproximadamente $40 \mathrm{~km}$ de altura) e uma resolução horizontal de $1.875^{\circ}$ por $1.25^{\circ}$, em longitude e latitude, respectivamente ( $192 \times 145$ pontos de grade), o que equivale a uma resolução cerca de $208 \mathrm{~km}$ por $139 \mathrm{~km}$ nas regiões tropicais.

Contém um modelo de vegetação dinâmico em que são considerados os ciclos de carbono oceânico e terrestre e a química da troposfera, incluindo aerossóis do tipo carbono orgânico e poeira. Processos bioquímicos oceânicos são representados pelo modelo HadOCC (Hadley Centre Ocean Carbon Cycle model).

\section{Resultados e Discussões}

\subsection{Avaliação do desempenho das simulações - Período Histórico}

A Tabela 5 apresenta os índices estatísticos obtidos para as três regiões de Moçambique, no período de dezembro de 1985 à novembro de 2005. Pode-se observar que os campos médios de velocidades de ventos simulados apresentaram correlações que vão de moderado a muito forte $(0,60<r<0,92)$ com os campos obtidos nos dois conjunto de reanálises. Sobre as regiões norte e centro encontra-se maiores valores do índice de concordância, sendo que para a região central esses valores são classificados de mediano a bom $(0,63<I c<0,72)$. Na região sul observa-se índice de concordância classificado como péssimo entre os campos simulados e as reanálises.

A Fig. 2 apresenta a comparação entre as séries climatológicas de ventos sobre a região norte de Moçambique. Os campos de ventos simulados apresentam padrões semelhantes aos apresentados nas reanálises, uma diferença no padrão é verificada no trimestre entre julho e setembro, em 
Tabela 4 - Configuração do modelo RAMS6.0.

\begin{tabular}{|c|c|c|}
\hline \multirow[t]{2}{*}{ Equações básicas } & \multicolumn{2}{|l|}{ Hidrostáticas incompreensíveis ou não compreensíveis. } \\
\hline & \multicolumn{2}{|l|}{ Não hidrostáticas de médias de Reynolds. } \\
\hline Coordenada vertical & \multicolumn{2}{|l|}{ Sigma-z, que segue a topografia (Clark, 1977) } \\
\hline Coordenada horizontal & \multicolumn{2}{|l|}{ Coordenada estereográfica polar rodada } \\
\hline Estrutura de grade & \multicolumn{2}{|l|}{ Arakawa-C (Mesinger E Arakawa, 1976) } \\
\hline \multirow[t]{9}{*}{ Física do modelo } & Esquema de adveção & Leapfrog \\
\hline & \multicolumn{2}{|l|}{ Avançado } \\
\hline & Turbulência & $\begin{array}{l}\text { Energia cinética turbulenta (Mellor e Yamada, 1974; } \\
\text { Deardoff, 1980) }\end{array}$ \\
\hline & \multirow[t]{2}{*}{ Convecção } & Kuo (1970) \\
\hline & & Kain-Fritsch (1990) \\
\hline & \multirow[t]{2}{*}{ Radiação } & Radiação de onda longa/curta (Mahrer e Pielke, 1977) \\
\hline & & Radiação de onda longa/curta(Harrington, 1997) \\
\hline & Microfísica das nuvens & Nuvens quentes (Walko et al., 1995; Meyer et al., 1997) \\
\hline & Solo e vegetação & Troca de calor entre solo e atmosfera (Walko, 2000) \\
\hline
\end{tabular}

Tabela 5 - Índices estatísticos calculados no período de dezembro de 1985 à novembro de 2005. Em que, b é o viés, EQM o erro quadrático médio, $r$ o coeficiente de correlação de Person e $I c$ o índice de concordância.

\begin{tabular}{|c|c|c|c|c|c|}
\hline \multicolumn{6}{|c|}{ Região Norte } \\
\hline & $b$ & $E Q M$ & $r^{*}$ & $R^{2}$ & $I_{c}$ \\
\hline RAMS/CFSR & 0,48 & 0,8 & 0,72 & 0,56 & 0,58 \\
\hline RAMS/MERRA & $-0,01$ & 0,89 & 0,61 & 0,39 & 0,53 \\
\hline \multicolumn{6}{|c|}{ Região Centro } \\
\hline & $b$ & $E Q M$ & $r^{*}$ & $R^{2}$ & $I_{c}$ \\
\hline RAMS/CFSR & 0,67 & 0,85 & 0,91 & 0,82 & 0,72 \\
\hline RAMS/MERRA & 0,39 & 0,9 & 0,78 & 0,62 & 0,63 \\
\hline \multicolumn{6}{|c|}{ Região Sul } \\
\hline & $b$ & $E Q M$ & $r^{*}$ & $R^{2}$ & $I_{c}$ \\
\hline RAMS/CFSR & 1,37 & 1,5 & 0,92 & 0,85 & 0,23 \\
\hline RAMS/MERRA & 1,12 & 1,43 & 0,8 & 0,65 & 0,18 \\
\hline
\end{tabular}

que as estimativas mostraram um aumento na velocidade do vento, padrão contrário ao apresentado pelas reanálises.

As correlações observadas entre aos campos médios simulados e os campos das reanálises do CFSR sobre a região norte vão de moderado à forte $(0,48<r<0,89)$, com exceção do mês de outubro em que a correlação obtida é classificada como fraca $(r<0,4)$. Em relação aos valores das reanálises do MERRA as simulações apresentaram correlações que vão de moderado à forte $(0,44<r<0,75)$, com exceção dos meses de outubro e novembro, em que a correlação novamente é classificada como fraca $(r<0,4)$.

Para a região central, os resultados mostraram que os campos simulados apresentaram padrões semelhantes aos dos campos de ventos obtidos nas reanálises, com exceção do período de janeiro a abril, em que as estimativas do modelo apresentam diminuição na velocidade média dos ventos, contrário aos padrões apresentados pelas reanálises. Observa-se que, em geral, os campos simulados superestimaram os valores das reanálises, com uma exceção no mês de abril, em que os campos de ventos simulados subestimaram as reanálises do MERRA.

As correlações estatísticas entre os valores da velocidade do vento simulados e os obtidos nas reanálises variam de forte a muito forte $(0,87<r<0,94)$ entre as séries simuladas e as reanálises do CFSR e são classificadas como fortes $(0,70<r<0,88)$ quando consideradas as reanálises do MERRA.

$\mathrm{Na}$ região sul de Moçambique observa-se que os padrões de campos de ventos simulados são semelhantes aos apresentados nas reanálises, com diferenças nos meses 

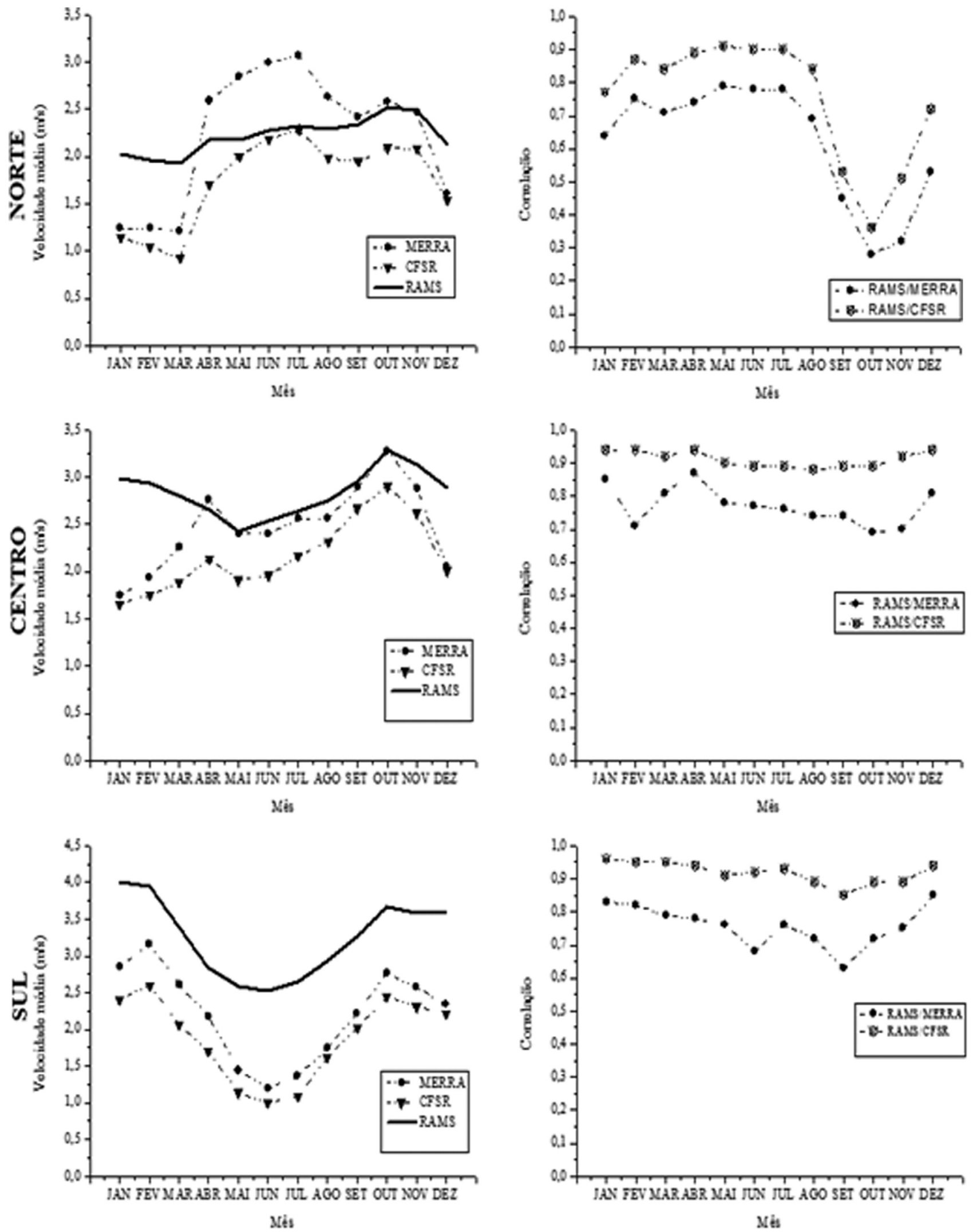

Figura 2 - Climatologia dos ventos simulados pelo modelo RAMS (esquerda) e correlações (direita). 
de janeiro e fevereiro, em que encontra-se estimativa de aumento na velocidade dos ventos simulados ao contrário do observado para este período nas séries de valores obtidos nas reanálises. Os resultados mostraram que os campos de ventos simulados superestimaram os campos de ventos das reanálises em todo o período.

As correlações entre as séries de valores médios da velocidade do vento simulados e as reanálises variam de moderado a muito forte $(0,64<r<0,95)$ na comparação com as reanálises do MERRA e de forte a muito forte na comparação com as reanálises do CFSR $(0,85<r<0,96)$. Porém, os altos valores encontrados nas correlações sugerem que os campos de ventos simulados apresentam um alto grau de associação com os campos de reanálises, mas que são quantitativamente diferentes.

\subsection{Impactos do cenário de mudanças climáticas (RCP8.5 - IPCC) sobre recursos eólicos de Moçambique}

A Tabela 6 apresenta os parâmetros de distribuição da velocidade dos ventos a $10 \mathrm{~m}$ de altura. Observa-se que a distribuição mensal de velocidade dos ventos, considerando-se o cenário emissão RCP8.5 (2079-2099), apresenta na maioria dos meses uma mediana positiva quando comparado com a mediana apresentada no período de 1985 2005. Isto sugere que os ventos irão soprar com velocidades máximas superiores às velocidades máximas do período de 1985-2005, bem como prevê-se maiores valores de velocidade média.

Na Tabela 7 tem-se os parâmetros de distribuição da velocidade dos ventos a $50 \mathrm{~m}$ de altura. Os resultados mostram que a distribuição mensal de velocidade dos ventos em condições do cenário emissão RCP8.5 (2079-2099) também apresenta na maioria dos meses uma mediana positiva comparando-se com a mediana apresentada no período histórico, que novamente sugere ventos com velocidades máximas superiores as obtidas no período histórico e maiores valores de velocidade média nessa altura.

A distribuição da velocidade dos ventos a $100 \mathrm{~m}$ de altura, mostrada na Tabela 8 , nesse cenário de emissões, concorda com as situações anteriores, ou seja, quando são comparados os campos de ventos nos dois períodos obtém-se, em geral, mediana positiva, indicando que os ventos devem apresentar velocidades médias e máximas superiores no período de 2079 a 2099.

Em relação a análise da direção do vento, apresentase os gráficos relativos as análises para $100 \mathrm{~m}$, altura média da instalação dos aerogeradores (Fig. 3). Os resultados demonstraram que de janeiro a abril do no período histórico os ventos predominantes são de sudeste (SE: 114 a $158^{\circ}$ ). Para o período de 2079-2099, a média do mês de janeiro mostra reduções em cerca de $7,0 \%, 34,0 \%$ e $10,0 \%$ na frequência média de direção dos ventos de leste-sudeste, sudeste e de sul-sudeste, respectivamente (Fig. 3b). Em fevereiro aparecem mudanças na frequência de direção dos ventos, com redução em cerca de $13,0 \%$ na frequência média dos ventos de sudeste, $18,0 \%$ na frequência média dos ventos de leste-sudeste (Fig. 3d).

No mês de março os ventos apresentam uma redução de $5,0 \%$ na frequência média de direção dos ventos de leste-sudeste, bem como aumento em cerca de $10,0 \%$ na frequência média de direção dos ventos de leste-sudeste, em relação aos padrões de direção dos ventos observados no período de 1985-2005. Para o mês de abril é previsto uma redução em cerca de $13,0 \%$ na frequência média de

Tabela 6 - Parâmetros da distribuição da velocidade dos ventos a $10 \mathrm{~m}$ de altura.

\begin{tabular}{|c|c|c|c|c|c|c|c|c|c|c|c|}
\hline \multirow[t]{3}{*}{ Mês } & \multicolumn{5}{|c|}{$1985-2005$} & \multicolumn{5}{|c|}{ 2079-2099 } & \multirow[t]{3}{*}{ Var_mediana (\%) } \\
\hline & \multicolumn{5}{|c|}{ Histórico } & \multicolumn{5}{|c|}{$\mathrm{RCP} 8.5$} & \\
\hline & $k$ & $c(\mathrm{~m} / \mathrm{s})$ & $v(\mathrm{~m} / \mathrm{s})$ & $\begin{array}{c}\text { Mediana } \\
(\mathrm{m} / \mathrm{s})\end{array}$ & $\begin{array}{c}\text { Desvpad } \\
(\mathrm{m} / \mathrm{s})\end{array}$ & $k$ & $c(\mathrm{~m} / \mathrm{s})$ & $v(\mathrm{~m} / \mathrm{s})$ & $\begin{array}{l}\text { Mediana } \\
(\mathrm{m} / \mathrm{s})\end{array}$ & $\begin{array}{c}\text { Desvpad } \\
(\mathrm{m} / \mathrm{s})\end{array}$ & \\
\hline Janeiro & 10,00 & 3,98 & 3,85 & 3,85 & 0,33 & 9,22 & 3,69 & 3,54 & 3,50 & 0,35 & $-9,09$ \\
\hline Fevereiro & 10,00 & 3,87 & 3,74 & 3,75 & 0,34 & 10,00 & 3,79 & 3,72 & 3,72 & 0,26 & $-0,80$ \\
\hline Março & 10,00 & 3,56 & 3,44 & 3,44 & 0,31 & 10,00 & 3,64 & 3,55 & 3,54 & 0,29 & 2,91 \\
\hline Abril & 10,00 & 3,47 & 3,35 & 3,32 & 0,31 & 10,00 & 3,60 & 3,50 & 3,49 & 0,27 & 5,12 \\
\hline Maio & 10,00 & 3,26 & 3,21 & 3,21 & 0,19 & 10,00 & 3,37 & 3,33 & 3,33 & 0,18 & 3,74 \\
\hline Junho & 10,00 & 3,45 & 3,38 & 3,38 & 0,22 & 10,00 & 3,52 & 3,43 & 3,48 & 0,28 & 2,96 \\
\hline Julho & 10,00 & 3,55 & 3,45 & 3,44 & 0,28 & 10,00 & 3,66 & 3,56 & 3,56 & 0,27 & 3,49 \\
\hline Agosto & 10,00 & 3,55 & 3,48 & 3,50 & 0,24 & 10,00 & 3,71 & 3,60 & 3,59 & 0,29 & 2,57 \\
\hline Setembro & 10,00 & 3,72 & 3,60 & 3,58 & 0,32 & 10,00 & 3,70 & 3,63 & 3,63 & 0,24 & 1,40 \\
\hline Outubro & 10,00 & 3,84 & 3,76 & 3,75 & 0,26 & 10,00 & 3,80 & 3,71 & 3,68 & 0,27 & $-1,87$ \\
\hline Novembro & 10,00 & 3,72 & 3,60 & 3,60 & 0,31 & 10,00 & 3,98 & 3,89 & 3,89 & 0,29 & 8,06 \\
\hline Dezembro & 10,00 & 3,61 & 3,47 & 3,43 & 0,32 & 10,00 & 3,59 & 3,52 & 3,51 & 0,24 & 2,33 \\
\hline
\end{tabular}


Tabela 7 - Parâmetros da distribuição da velocidade dos ventos a $50 \mathrm{~m}$ de altura.

\begin{tabular}{|c|c|c|c|c|c|c|c|c|c|c|c|}
\hline \multirow[t]{3}{*}{ Mês } & \multicolumn{5}{|c|}{$1985-2005$} & \multicolumn{5}{|c|}{ 2079-2099 } & \multirow[t]{3}{*}{ Var_mediana $(\%)$} \\
\hline & \multicolumn{5}{|c|}{ Histórico } & \multicolumn{5}{|c|}{ RCP8.5 } & \\
\hline & $k$ & $c(\mathrm{~m} / \mathrm{s})$ & $v(\mathrm{~m} / \mathrm{s})$ & $\begin{array}{c}\text { Mediana } \\
(\mathrm{m} / \mathrm{s})\end{array}$ & $\begin{array}{c}\text { Desvpad } \\
(\mathrm{m} / \mathrm{s})\end{array}$ & $k$ & $c(\mathrm{~m} / \mathrm{s})$ & $v(\mathrm{~m} / \mathrm{s})$ & $\begin{array}{c}\text { Mediana } \\
(\mathrm{m} / \mathrm{s})\end{array}$ & $\begin{array}{c}\text { Desvpad } \\
(\mathrm{m} / \mathrm{s})\end{array}$ & \\
\hline Janeiro & 4,66 & 2,93 & 2,68 & 2,63 & 0,65 & 4,30 & 2,83 & 2,58 & 2,56 & 0,67 & $-2,66$ \\
\hline Fevereiro & 5,35 & 3,06 & 2,82 & 2,77 & 0,59 & 5,46 & 3,21 & 2,96 & 2,97 & 0,60 & 7,22 \\
\hline Março & 5,97 & 3,53 & 3,27 & 3,26 & 0,62 & 6,54 & 3,91 & 3,65 & 3,65 & 0,62 & 11,96 \\
\hline Abril & 6,22 & 3,95 & 3,68 & 3,67 & 0,65 & 7,27 & 4,15 & 3,90 & 3,88 & 0,58 & 5,72 \\
\hline Maio & 6,08 & 3,41 & 3,17 & 3,18 & 0,59 & 6,47 & 3,60 & 3,36 & 3,33 & 0,58 & 4,72 \\
\hline Junho & 6,25 & 3,69 & 3,44 & 3,42 & 0,57 & 10,00 & 3,52 & 3,43 & 3,48 & 0,28 & 1,75 \\
\hline Julho & 6,34 & 3,75 & 3,49 & 3,52 & 0,64 & 5,52 & 3,83 & 3,54 & 3,52 & 0,69 & 0,00 \\
\hline Agosto & 5,87 & 3,63 & 3,37 & 3,38 & 0,65 & 5,52 & 4,01 & 3,71 & 3,68 & 0,73 & 8,88 \\
\hline Setembro & 5,40 & 3,95 & 3,65 & 3,61 & 0,75 & 6,14 & 4,02 & 3,74 & 3,66 & 0,67 & 1,39 \\
\hline Outubro & 6,27 & 4,17 & 3,88 & 3,79 & 0,69 & 6,76 & 4,36 & 4,08 & 4,01 & 0,66 & 5,80 \\
\hline Novembro & 6,02 & 4,29 & 3,98 & 3,91 & 0,73 & 6,25 & 4,38 & 4,07 & 4,10 & 0,73 & 4,86 \\
\hline Dezembro & 4,65 & 3,05 & 2,79 & 2,66 & 0,66 & 4,99 & 3,48 & 3,19 & 3,10 & 0,71 & 16,54 \\
\hline
\end{tabular}

Tabela 8 - Parâmetros da distribuição da velocidade dos ventos a 100 m.

\begin{tabular}{|c|c|c|c|c|c|c|c|c|c|c|c|}
\hline \multirow[t]{3}{*}{ Mês } & \multicolumn{5}{|c|}{$1985-2005$} & \multicolumn{5}{|c|}{ 2079-2099 } & \multirow[t]{3}{*}{ Var_mediana $(\%)$} \\
\hline & \multicolumn{5}{|c|}{ Histórico } & \multicolumn{5}{|c|}{ RCP8.5 } & \\
\hline & $k$ & $c(\mathrm{~m} / \mathrm{s})$ & $v(\mathrm{~m} / \mathrm{s})$ & $\begin{array}{c}\text { Mediana } \\
(\mathrm{m} / \mathrm{s})\end{array}$ & $\begin{array}{c}\text { Desvpad } \\
(\mathrm{m} / \mathrm{s})\end{array}$ & $k$ & $c(\mathrm{~m} / \mathrm{s})$ & $v(\mathrm{~m} / \mathrm{s})$ & $\begin{array}{c}\text { Mediana } \\
(\mathrm{m} / \mathrm{s})\end{array}$ & $\begin{array}{c}\text { Desvpad } \\
(\mathrm{m} / \mathrm{s})\end{array}$ & \\
\hline Janeiro & 4,55 & 3,17 & 2,89 & 2,85 & 0,72 & 4,24 & 3,02 & 2,75 & 2,72 & 0,73 & $-4,56$ \\
\hline Fevereiro & 5,23 & 3,32 & 3,05 & 3,04 & 0,64 & 5,08 & 3,39 & 3,12 & 3,11 & 0,70 & 2,30 \\
\hline Março & 5,83 & 3,87 & 3,58 & 3,57 & 0,69 & 6,30 & 4,19 & 3,91 & 3,88 & 0,68 & 8,68 \\
\hline Abril & 6,05 & 4,37 & 4,06 & 4,00 & 0,74 & 6,94 & 4,47 & 4,19 & 4,14 & 0,65 & 3,50 \\
\hline Maio & 5,77 & 3,80 & 3,52 & 3,52 & 0,69 & 6,11 & 3,90 & 3,63 & 3,61 & 0,66 & 2,56 \\
\hline Junho & 6,08 & 4,11 & 3,84 & 3,79 & 0,65 & 5,78 & 3,51 & 3,24 & 3,28 & 0,65 & $-13,46$ \\
\hline Julho & 6,14 & 4,20 & 3,90 & 3,93 & 0,73 & 5,37 & 4,19 & 3,87 & 3,83 & 0,78 & $-2,54$ \\
\hline Agosto & 5,62 & 4,11 & 3,80 & 3,78 & 0,77 & 5,29 & 4,38 & 4,04 & 4,03 & 0,83 & 6,61 \\
\hline Setembro & 5,15 & 4,48 & 4,12 & 4,09 & 0,89 & 5,82 & 4,38 & 4,06 & 3,95 & 0,77 & $-3,42$ \\
\hline Outubro & 5,65 & 4,74 & 4,38 & 4,31 & 0,86 & 6,30 & 4,75 & 4,42 & 4,42 & 0,78 & 2,55 \\
\hline Novembro & 5,13 & 4,31 & 3,96 & 3,91 & 0,85 & 5,86 & 4,73 & 4,38 & 4,46 & 0,84 & 14,07 \\
\hline Dezembro & 4,44 & 3,37 & 3,07 & 2,92 & 0,76 & 4,79 & 3,75 & 3,43 & 3,32 & 0,80 & 13,70 \\
\hline
\end{tabular}

direção dos ventos de leste-sudeste, assim como prevê-se um aumento de $10,0 \%$ na frequência dos ventos de sudeste.

A continuação da Fig. 3 apresenta a distribuição da direção dos ventos para os meses de maio a agosto. Ao compararmos os padrões de direção dos ventos para o mês de maio, no período de 2079-2099 (Fig. 3j), observa-se um aumento estimado em $11,0 \%$ na frequência média de direção apenas dos ventos de sudeste. Em relação ao mês de junho, prevê-se um aumento estimado de $18,0 \%$ na frequência média de direção nos ventos de sudeste (Fig. 31).

Os padrões de direção de ventos para o mês de julho, indicam um aumento em cerca de $26,0 \%$ da frequência média apenas dos ventos de sudeste (Fig. 3n). No mês de agosto observam-se mudanças na frequência de direção dos ventos, com aumento em cerca de $10,0 \%$ na frequência média dos ventos de sudeste, bem como uma redução em cerca de $25,0 \%$ na frequência média de direção dos ventos de leste-sudeste em relação a frequência média de direção dos ventos para o período de 1985-2005.

Ainda em continuação a Fig. 3, apresenta-se a distribuição da direção dos ventos para os meses de setembro a dezembro. Os resultados mostraram que no mês de setembro são observados ventos predominantemente de lestesudeste (ESE: $112,5^{\circ}$ ) (Fig. 3q), com um aumento de 4,0\% 

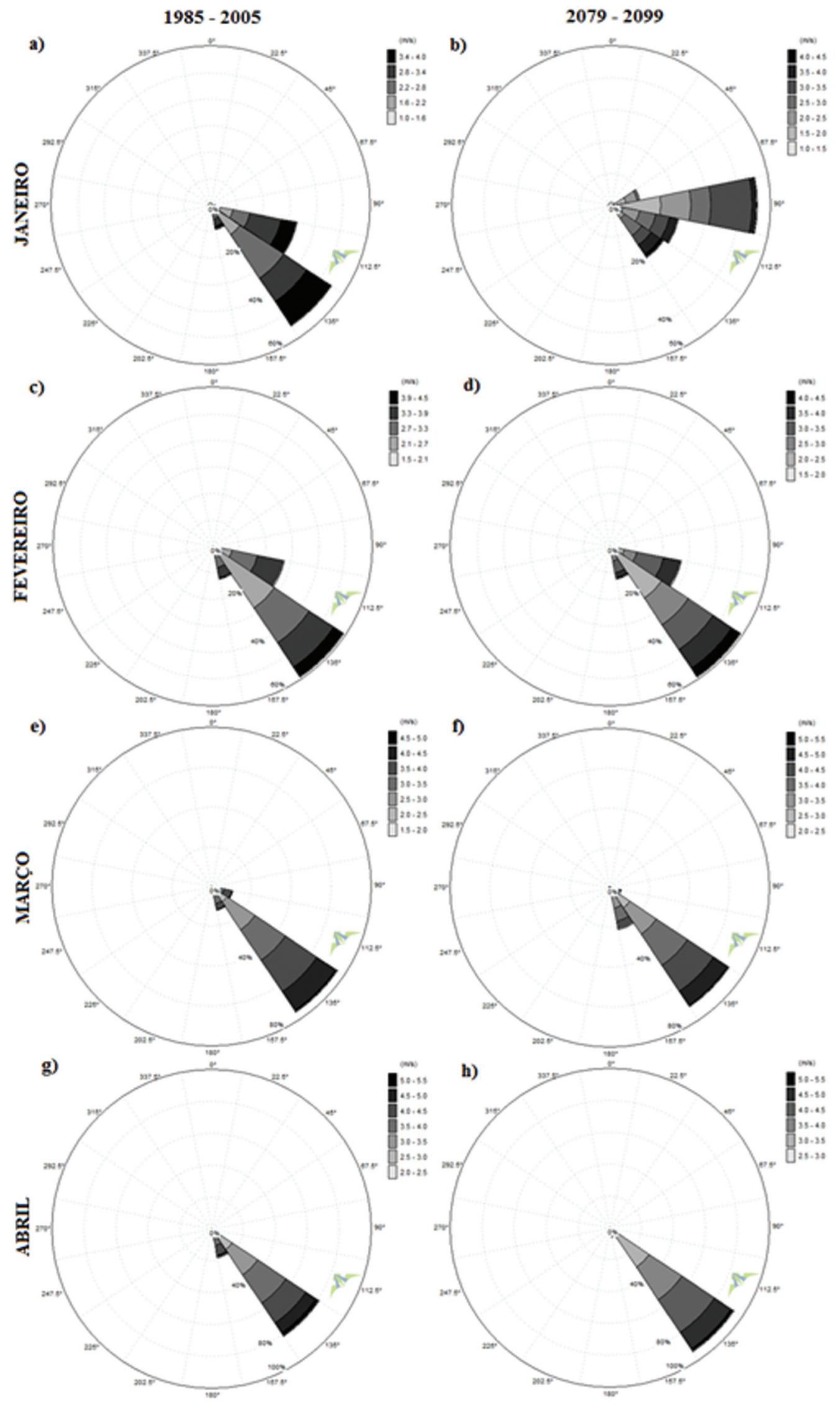

Figura 3 - Mapas de direção dos ventos a $100 \mathrm{~m}$. 

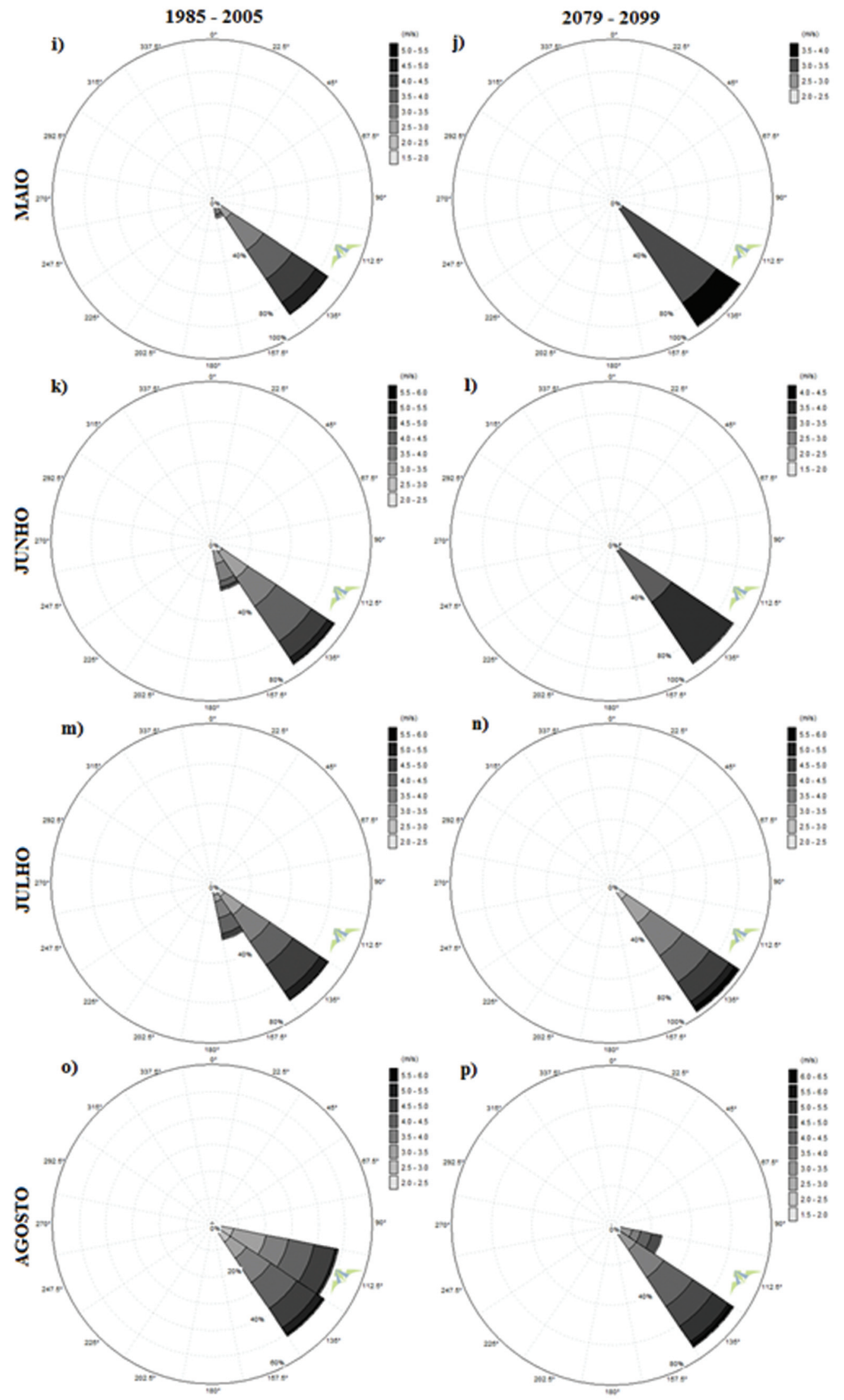

Figura 3 - Cont. 


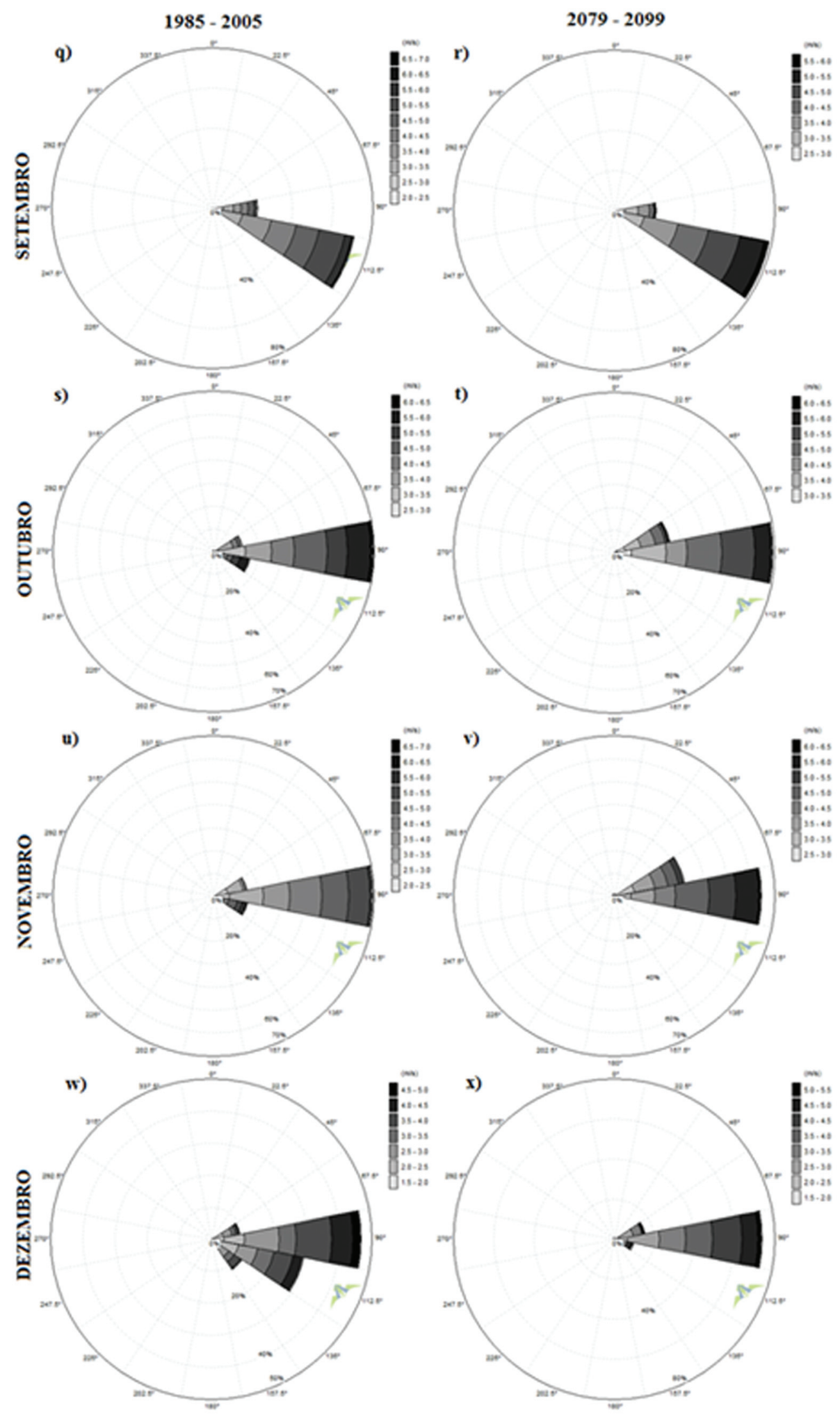

Figura 3 - Cont. 
na frequência média nesta direção no período de 2079-2099 (Fig. 3r). No mês de outubro estima-se um aumento de $13,0 \%$ na frequência média de direção apenas dos ventos de leste-nordeste.

Ao compararmos os padrões de direção dos ventos para o mês de novembro, observa-se uma ausência prevista para os ventos de leste-sudeste para o período de 20792099. Como também, uma redução de 5,0\% na frequência média de direção dos ventos de leste e um aumento de $16 \%$ na frequência de direção dos ventos de leste-nordeste (Fig. 3v).

Para o mês de dezembro, tem-se previsão de aumento na frequência média em cerca de $6,0 \%$ na direção dos ventos de leste-nordeste, de $25 \%$ na direção dos ventos de leste, e redução de $20 \%$ na frequência média de direção dos ventos de leste-sudeste (Fig. 3x).

A análise da densidade de potência média $\left(\mathrm{W} / \mathrm{m}^{2}\right)$ a $100 \mathrm{~m}$ de altura é apresentada na Fig. 4. Os resultados mostram que no trimestre de dezembro a fevereiro (DJF) a maior parte do país apresenta uma densidade de potência média estimada de $40-80 \mathrm{~W} / \mathrm{m}^{2}$, porém em algumas áreas na região centro-oeste estima-se uma densidade de potência média superior a $100 \mathrm{~W} / \mathrm{m}^{2}$. Para o período de 2079-2099 são esperadas mudanças na densidade de potência, de tal forma que se prevê um aumento de $25,5 \%$ sobre a região norte, de $19,1 \%$ sobre a região centro e de $22,4 \%$ sobre a região sul.

No trimestre de março a maio (MAM), em algumas porções de áreas sobre a região centro-oeste apresentam uma estimativa de densidade de potência média acima dos $120 \mathrm{~W} / \mathrm{m}^{2}$. Para o período de 2079-2099 tem-se a previsão de mudanças na densidade de potência média, com reduções de $15,5 \%$ sobre a região norte e de $2,7 \%$ sobre a região centro.

$\mathrm{Na}$ trimestre de julho a agosto (JJA), as regiões centro e sul apresentam estimativas de densidade de potência média, com valores entre $40-80 \mathrm{~W} / \mathrm{m}^{2}$. Com previsão de mudanças para o período de 2079-2099, aumento de 14,8\% sobre a região centro e redução de $6,8 \%$ na densidade de potência média sobre a região sul.

Por fim, no trimestre de setembro a novembro (SON) tem-se que a maior parte do país apresenta uma densidade de potência média estimada que chega a $80 \mathrm{~W} / \mathrm{m}^{2}$. Para o período de 2079-2099 é esperado o aumento de 66,8\% sobre a região norte, aumento de $8,5 \%$ sobre a região centro e uma redução de $20,4 \%$ sobre a região sul.

\section{Conclusões}

A comparação das simulações dos campos dos ventos com os campos obtidos das reanálises do CFSR e MERRA mostraram que, com o método empregado, o modelo RAMS foi capaz de reproduzir os padrões médios dos campos dos ventos obtidos nas reanálises, que são em alguns casos considerados como observações, embora algumas diferenças de padrões tenha sido encontradas nas regiões centro e sul do país nos meses de novembro e março.

As correlações espaciais entre os campos dos ventos simulados mostraram, em geral, um alto grau de correlação com os campos dos ventos das reanálises. Maiores erros das estimativas foram encontrados na comparação dos valores simulados com os das reanálises para a região Sul de Moçambique e, consequentemente, baixos índices de concordância entre as séries de valores simulados e das reanálises adotadas na comparação. Evidenciando, assim, uma diminuição do desempenho das simulações nessa região.

O estudo mostrou que os recursos eólicos esperados no futuro para o período de 2079-2099 sobre a região de Moçambique não estão uniformemente distribuídos. Além disso, verificam-se mudanças na velocidade dos ventos, bem como mudanças na densidade de potência eólica sobre a área de estudo. Os resultados sugerem um aumento médio sobre a média anual de velocidade dos ventos estimado entre 2,0 a $4,0 \%$ para ventos a $10 \mathrm{~m}$ de altura, aumento estimado entre 2,0 a 9,0\% para ventos a $50 \mathrm{~m}$ de altura e um aumento estimado entre 1,5 a $7,0 \%$ para ventos a $100 \mathrm{~m}$ de altura.

A análise com objetivo de determinar as possíveis mudanças na direção e distribuição de velocidades dos ventos calculadas para o período de 1985-2005 e comparadas com o período de 2079-2099, para o cenário de emissão do RCP8.5, indicaram não haver evidências de mudanças significativas nos padrões de direção dos ventos, no entanto, prevê-se mudanças na frequência dos ventos em algumas determinadas direções, além disso os resultados mostraram a predominância dos ventos de sudeste nos dois períodos de análise.

As simulações de campos de ventos sob cenário de emissão RCP8.5 mostraram um aumento nos parâmetros de distribuição de velocidade dos ventos, o que é provável que as velocidades para o período de 2079-2099 apresentem maiores velocidades de velocidade média bem como apresentem valores de velocidade máximas superiores às observadas no período de 1985-2005.

Por fim, este trabalho mostrou que são previstas mudanças na velocidade dos ventos e consequentemente mudanças na densidade de energia eólica sobre a região de Moçambique para o período de 2079-2099. Todavia, para maior aprofundamento do entendimento dos impactos das mudanças climáticas nos recursos eólicos sobre a região de Moçambique, indica-se a realização de investigações nessa linha de raciocínio para os períodos de 2015-2035 e 20452065 , que não foram investigados em virtude da alta demanda de recursos computacionais na realização das simulações climáticas. 

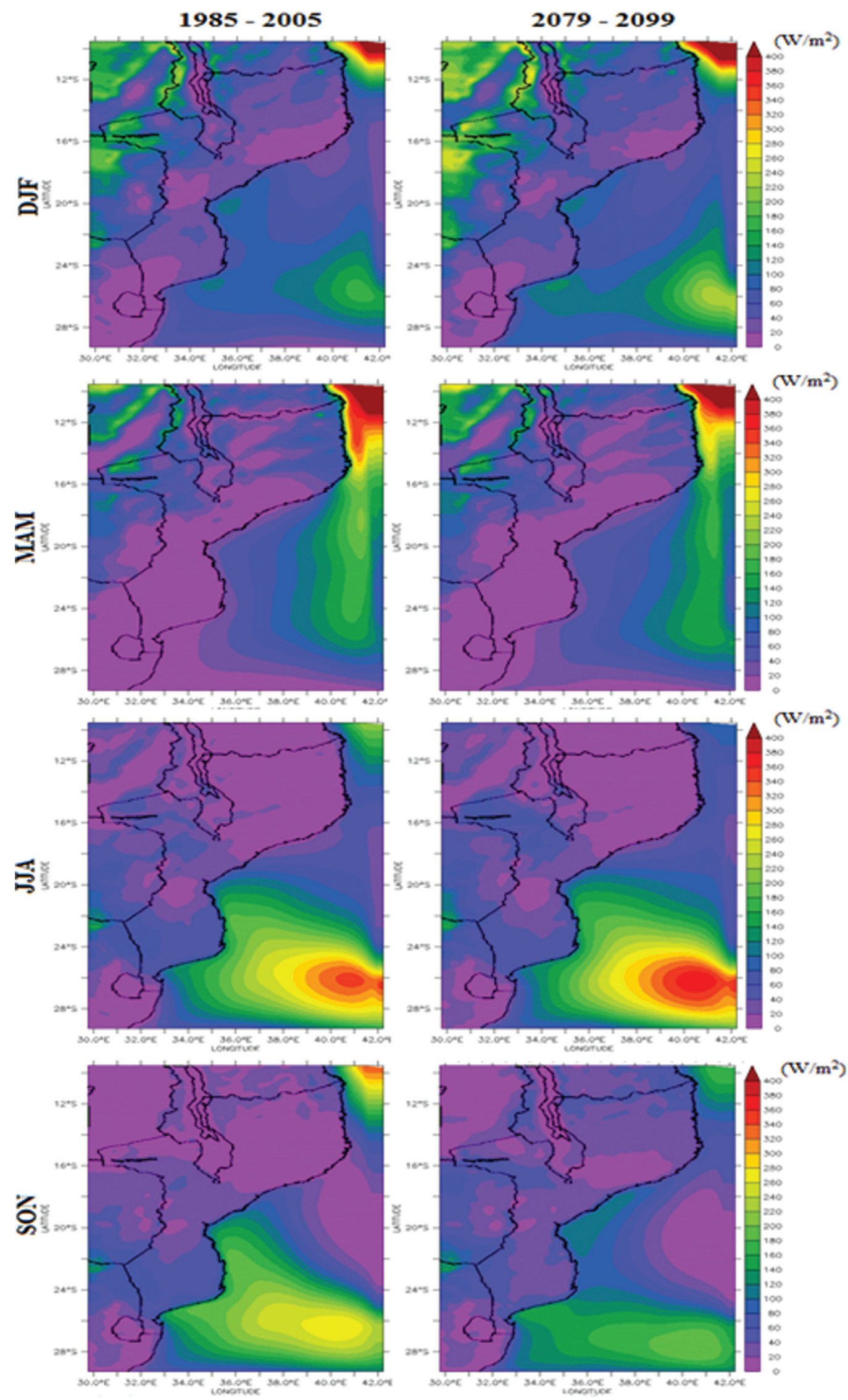

Figura 4 - Mapas de densidade de potência média a 100 m (W/m²), períodos: Histórico (1985-2005) e Futuro (2079-2099). 


\section{Referências}

BRESLOW P.B. and SAILOR D.J. Vulnerability of Wind Power Resources to Climate Change in the Continental United States. Renewable Energy, v. 27, n. 4, p. 585-598, 2002.

BOSILOVICH, M.G.J.; CHEN, F.R. ROBERTSON, and ADLER, R.F. Evaluation of global precipitation in reanalyses. Journal of Applied Meteorology and Climatology, v. 47, n. 1, p. 2279-2299, 2008.

BUYS, P.; DEICHMANN U., MEISNER C., TON-THAT T. and WHEELER D. Country Stakes in Climate Change Negotiations: Two Dimensions of Vulnerability. Policy Research Working Paper 4300 - World Bank, v. 1, n. 1, p. 95, 2007.

COTTON, W.R.; PIELKE, R.A.; WALKO, R.L.; LISTON G.E.; TREMBACK, C.J.; JYAN, H.; MC ANELLY, R.L.; HARRINGTON, J.Y.; NICHOLLS, M.E.; CARRIO, C.P.; ANDERSSON, E.; HECKLEY, W.; PAILLEUX, J.; VASILJEVIC, D.; HAMRUD, M.; HOLLINGSWORTH, A.; RABIER, F.; FISHER, M. The ECMWF implementation of three dimensional variational assimilation 3D-Var. Part I: Formulation. Quarterly Journal of the Royal Meteorological Society, v. 124, n. 1, p. 1783-1808, 1998.

DEVORE, J.L. Probabilidade e Estatística para Engenharia e $\mathrm{Cie}^{\wedge}$ ncias. Pioneira Thomson Learning, São Paulo, 2006.

HARRISON, G.P., CRADDEN, L.C., CHICK, J.P. Preliminary assessment of climate change on the UK onshore wind energy resource. Energy Sources, v. 30, n.14, p. 1286-1299, 2008.

HUANG, G.; PAES, A.T. Posso usar o teste t de Student quando preciso comparar três ou mais grupos? Einstein: Educação Continuada em Saúde. v. 7, n. 2, p. 63-64, 2009.

GWEC (Global Wind Energy Council). Global wind statistics. 2012.

MENEZES, H.E.A.; BRITO, J.I.B.; SANTOS, C.A.C.; SILVA, L.L. A relação entre a temperatura da superfície dos oceanos tropciais e a duração dos veranicos nos Estado da Paraíba. Revista Brasileira de Meteorologia, v. 23, n. 2, p. 152-161, 2008.
NOLAN, P.; LYNCH, P.; McGRATH, R.; SEMMLER, T. and WANG, S. Simulating Climate Change and its Effects on the Wind Energy Resource of Ireland. Wind Energy, v. 15, n. 1, p. 593-608, 2012.

OLIVEIRA, C.E.L.; FRÜHLING, I.; URIBE-OPAZO, M.A. Análise climatológica do potencial eólico na região de Cascavel - PR. Engenharia Agrícola - Jaboticabal, v. 23, n. 3, p. 425-433, 2003.

PIELKE, R.A.R.; COTTON W.R.; WALKO R.L.; TREMBACK C.J.; LYONS W.A.; GRASSO L.D.; NICHOLLS M.E.; MORAN M.D.; WESLEY D.A.; LEE T.J. and COPELAND J.H.A. Comprehensive Meteorological Modeling System RAMS. Meteorology and Atmospheric Physics. v. 49, n. 1, p. 69-91, 1992.

PINTO, I. Elaboração de um Atlas de Ventos para Moçambique usando o modelo Regional do Clima RegCM. Trabalho de licenciatura. Moçambique, 2008.

PRYOR S.C.; BARTHELMIE R.J.; KJELLSTRO, M.E. Potential Climate Change Impact on Wind Energy Resources in Northern Europe: Analyses Using a Regional Climate Model. Climate Dynamics, v. 25, n. 7, p. 815-835, 2005.

SAILOR D.J.; SMITH, M. and HEART, M. Climate Change Implications for Wind Power Resources in the Northwest United States. Renewable Energy, v. 33, n. 11, pp. 2393-2406, 2008.

SANTIAGO DE MARIA, P.H.; COSTA, A.A.; SOMBRA, S.S. Modelagem numérica em alta resolução para previsão de geração de energia eólica no Ceará. Revista Brasileira de Meteorologia, v. 23, n. 4, p. 477-489, 2008.

SEGAL, M.; PAN Z.; ARRITT, R.W. and TAKE, E.S. On the Potential Change in Wind Power over the US Due to Increases of Atmospheric Greenhouse Gases. Renewable Energy, v. 24, n. 2, p. 235-243, 2001.

TREMBACK, C.J.; WALKO, R.L. RAMS - Version 6.0: User's guide - Introduction. Fort Collins (CO), EUA, 2005.

WILLMOTT, C.J. On the validation of models. Physical Geography, v. 2, n. 2, p. 184-194, 1981.

This is an Open Access article distributed under the terms of the Creative Commons Attribution Non-Commercial License which permits unrestricted non-commercial use, distribution, and reproduction in any medium provided the original work is properly cited. 